\title{
BMJ Open Incremental healthcare utilisation and costs among new senior high-cost users in Ontario, Canada: a retrospective matched cohort study
}

\author{
Sergei Muratov, ${ }^{1}$ Justin Lee, ${ }^{2}$ Anne Holbrook, ${ }^{3}$ Jason Robert Guertin, ${ }^{4}$ \\ Lawrence Mbuagbaw (10 ,5 John Michael Paterson, ${ }^{6}$ Tara Gomes, ${ }^{6,7}$ \\ Priscila Pequeno, ${ }^{6}$ Jean-Eric Tarride ${ }^{8}$
}

To cite: Muratov S, Lee J, Holbrook A, et al. Incremental healthcare utilisation and costs among new senior high-cost users in Ontario, Canada: a retrospective matched cohort study. BMJ Open 2019;9:e028637. doi:10.1136/ bmjopen-2018-028637

- Prepublication history and additional material for this paper are available online. To view these files, please visit the journal online (http://dx.doi. org/10.1136/bmjopen-2018028637).

Received 17 December 2018 Revised 31 August 2019 Accepted 05 September 2019

Check for updates

(C) Author(s) (or their employer(s)) 2019. Re-use permitted under CC BY-NC. No commercial re-use. See rights and permissions. Published by BMJ.

For numbered affiliations see end of article.

Correspondence to

Sergei Muratov;

muratos@mcmaster.ca

\section{ABSTRACT}

Objectives To describe healthcare use and spending before and on becoming a new (incident) senior high-cost user (HCU) compared with senior non-HCUs; to estimate the incremental costs, overall and by service category, attributable to HCU status; and to quantify its monetary impact on the provincial healthcare budget in Ontario, Canada.

Design We conducted a retrospective, population-based comparative cohort study using administrative healthcare records. Incremental healthcare utilisation and costs were determined using the method of recycled predictions allowing adjustment for preincident and incident year values, and covariates. Estimated budget impact was computed as the product of the mean annual total incremental cost and the number of senior HCUs. Participants Incident senior HCUs were defined as Ontarians aged $\geq 66$ years who were in the top $5 \%$ of healthcare cost users during fiscal year 2013 (FY2013) but not during FY2012. The incident HCU cohort was matched with senior non-HCUs in a ratio of $1 \mathrm{HCU}: 3$ non-HCU. Results Senior HCUs ( $n=175847)$ reached the annual HCU threshold of CAD\$10 192 through different combinations of incurred costs. Although HCUs had higher healthcare utilisation and costs at baseline, HCU status was associated with a substantial spike in both, with prolonged hospitalisations playing a major role. Twelve per cent of HCUs reached the HCU expenditure threshold without hospitalisation. Compared with non-HCUs ( $n=527$ 541), HCUs incurred an additional CAD $\$ 25527$ per patient in total healthcare costs; collectively CAD $\$ 4.5$ billion or $9 \%$ of the 2013 Ontario healthcare budget. Inpatient care had the highest incremental costs: CAD $\$ 13427,53 \%$ of the total incremental spending.

Conclusions Costs attributable to incident senior HCU status accounted for almost $1 / 10$ of the provincial healthcare budget. Prolonged hospitalisations made a major contribution to the total incremental costs. A subgroup of patients that became HCU without hospitalisation requires further investigation.

\section{INTRODUCTION}

Healthcare spending has more than doubled in the countries of the Organisation for
Strengths and limitations of this study

- This population-based study examines incident (new) senior high-cost users (HCUs), which provides important information on the driving factors for $\mathrm{HCU}$ status.

> Inclusion of all incident senior HCU in the province into the study population allowed us to calculate their monetary impact on the provincial healthcare budget.

- This analysis includes a comprehensive spectrum of the most important cost categories that contribute to total public healthcare expenditures in the province.

- Despite the comprehensiveness of cost analyses, a few of the cost categories may not have been captured in full, for example, outpatient intravenous chemotherapy.

- The findings, especially with respect to the total incremental costs and the budget impact, are only comparable to studies with the same HCU threshold and the choice of cost categories.

Economic Co-operation and Development over the past two decades. ${ }^{1}$ In Canada, where public health and healthcare are under provincial jurisdiction, health spending accounts for $37 \%$ of the total provincial programme spending on average. ${ }^{2}$ Much of the spending is disproportionately attributed to a small but heterogeneous group of patients, commonly referred to as high-cost healthcare users (HCUs). ${ }^{3-5}$ The pressing need to control healthcare spending and the inconclusive evidence and varying success of clinical interventions targeting the HCU group $^{67}$ have prompted policy-makers to revise their management strategies and to seek specific segments of the HCU population who may benefit from certain interventions more than others. ${ }^{489}$

Incident (or new) senior HCUs represent one such segment whose patient care 
characteristics and spending patterns have not been well studied. A recent systematic review identified 55 studies published over the past two decades that reported HCU characteristics and healthcare utilisation. ${ }^{5}$ The vast majority $(n=42)$ of the publications originated from the USA, nine were from Canada, three were generated by researchers from European countries and one was from Taiwan. Compared with nine US-based studies of the Medicare (ie, senior) population, only the study from Taiwan among the others had a specific focus on seniors, even though approximately $45 \%-55 \%$ of senior healthcare care resources are reportedly consumed by senior HCUs in various jurisdictions. ${ }^{10-12}$ Moreover, these studies do not differentiate between prevalent (who retain the HCU status over years) and incident senior HCUs. This is important, as understanding the path to HCU status may identify opportunities for intervention. ${ }^{4}$ Further, it is well known that senior HCUs, both prevalent and incident, generally have poor functional status and consume a high level of healthcare resources, including typically reported acute inpatient care and physician services. ${ }^{73} 14$ However, comprehensive descriptions of cost drivers to HCU status are few. ${ }^{10}{ }^{15}$ A recent example is a study conducted in Ontario, the largest province in Canada, which presented a system-wide assessment of cost concentration among HCUs over 3 years using both longitudinal and cross-sectional approaches to their analysis. ${ }^{10}$ While providing valuable information on the transition of patients between various cost strata, their longitudinal analyses focused on the persistence of costs among all HCUs. Their cross-sectional analysis of expenditures by cost category was limited by only reporting on the top $1 \%$ of HCUs and was not stratified by age. Another poorly explored aspect of HCU cost analysis is the economic burden associated with HCU status, which remains largely unknown in Canada and elsewhere. While some international studies have compared costs between HCUs and non-HCU cohorts in a particular year using a cross-sectional design, ${ }^{3} 1617$ these comparative studies did not consider any secular trends over time (eg, costs in the years before the incident year). This limits our understanding of the true incremental costs of becoming a new HCU, especially among seniors.

We recently reported on a cohort of incident senior HCUs compared with matched non-HCUs to examine regional variation in mortality and costs in Ontario using cross-sectional data. ${ }^{18}$ Here, we aim to determine the incremental healthcare utilisation and costs among new senior HCUs in Ontario by looking at the same data longitudinally. The main objectives of this study were to (1) describe healthcare use and spending before and on becoming a senior HCU compared with senior non-HCUs; (2) estimate costs and healthcare use attributable to the incident senior HCU status and to (3) quantify the monetary impact of incident senior HCUs on the provincial healthcare budget.

\section{METHODS}

\section{Study design}

We conducted a retrospective population-based matched cohort study using administrative healthcare data from Ontario, Canada. The protocol for this research has been published. ${ }^{19}$

\section{Setting and data sources}

Ontario is Canada's most populous province, with almost 14 million residents (approximately $40 \%$ of the Canadian population).$^{20}$ The Ontario Ministry of Health and LongTerm Care (MOHLTC) pays for approximately $70 \%$ of healthcare provided in the province. This includes nearly $100 \%$ of hospital care, physician services and prescription drugs for seniors. ${ }^{21}$

A patient-level dataset was created by linking 19 health administrative databases ${ }^{19}$ using unique encoded identifiers at Institute for Clinical Evaluative Sciences (ICES) (www.ices.on.ca). ICES is an independent, non-profit research corporation funded by the Ontario MOHLTC. The Ontario government fiscal year 2013 (1 April 2013 and 31 March 2014) was considered the incident year (FY2013). FY2012: 1 April 2012 and 31 March 2013 was the baseline or preincident year.

\section{Study population}

Incident senior HCUs were defined as individuals aged 66 years or above with annual total healthcare expenditures in the top 5\% of all Ontarians in FY2013, who were not in the top 5\% healthcare users in FY2012. The 5\% threshold is commonly reported in HCU studies in Canada and elsewhere. ${ }^{10142223}$ The $>66$ years age threshold was applied to capture Ontario Drug Benefit (ODB) expenditures for at least 1 year before the incident year: ODB coverage starts automatically when Ontarians reach 65 years of age. $^{24}$ The 'non-HCU' cohort included those whose annual total healthcare expenditures in FY2012 and FY2013 were below the top 5\% threshold in both years. The incident HCU cohort was matched with non-HCU in a ratio of $1 \mathrm{HCU}: 3$ non-HCUs by age at the cohort entry (within 1 month), sex and Local Health Integration Network (LHIN) of patient residence. LHINs, Ontario's 14 regional health districts, are responsible for the planning and administration of most of hospital-based and community-based health services delivered within their geographical boundaries. ${ }^{25}$

\section{Variables}

Our dataset included key information on sociodemographic and health status, healthcare utilisation and costs. Described in the study protocol ${ }^{19}$ in more detail, key variables are briefly summarised below.

Sociodemographic status included age, sex, low income status and geography of residence (urban/suburban/ rural). Low-income status was based on net household income reported to receive ODB subsidy in FY2012. Rurality was based on the Rurality Index for Ontario (RIO), which is a scale from 0 to 100 . An RIO between 0 
and 9 defined an individual from the urban area, between 10 and 40 described a suburban resident, and a resident from a rural area had an RIO score of 40 and above. ${ }^{26}$

Health status was assessed using several variables. We used two tools derived from Johns Hopkins Adjusted Clinical Groups (ACGs) System, V.10, a casemix methodology to describe a population's healthcare utilisation looking back for 3 years prior to the incident year. ${ }^{27}$ First, the general degree of comorbidity was captured by the number of Johns Hopkins Aggregated Diagnosis Groups (ADGs): person-focused, diagnosis-based method to measure patients' illness by assigning individual ACGs into diagnosis clusters. ${ }^{28} \mathrm{~A}$ higher number of ADGs per patient indicates a greater burden of illness. In addition, we identified the proportion of patients with a history of hypertension, malignancy and mental health condition using John Hopkins Expanded Diagnosis Clusters. For each condition, we checked whether the patient was diagnosed with the condition in the 3 years prior to FY2013. Finally, we used validated administrative data case definitions to identify whether the patient had a history of several common chronic diseases, including congestive heart failure, diabetes and chronic obstructive pulmonary disease. ${ }^{29}{ }^{30}$ The choice of specific conditions used to describe patients was driven by several factors: (1) chronic conditions that are commonly associated with high economic burden (cardiovascular and pulmonary diseases, malignancy) ${ }^{31-33} ;(2)$ conditions that are well known risk factors (eg, hypertension, diabetes) and (3) availability of data.

Whereas sociodemographic characteristics and health status were captured at baseline, healthcare utilisation and expenditures were obtained for the full 2 years of study. Utilisation variables included the number of hospitalisations (all, elective and unplanned), emergency department (ED) visits, physician encounters and publicly funded home care services. Home care services were subclassified by type of service: nursing, personal support and allied health. For each hospitalisation, we obtained the total length of stay (TLOS), in days.

Healthcare expenditures were estimated using ICES person-level health utilisation costing algorithms, ${ }^{34}$ which report expenditures according to 12 health service cost categories. Hospital costs were the sum of costs associated with acute inpatient care and same-day surgery. Mental health admissions were costed separately. Physician expenditures were the sum of fee-for-service billings and capitation payments. The cost categories also separately included publicly funded long-term homes, inpatient rehabilitation services, community home care and admissions to complex continuing care. Costs were expressed in 2013 Canadian Dollars.

\section{Outcomes}

The primary outcome measures were (1) 1-year incremental healthcare utilisation for hospital admissions (total and by types such as unplanned and elective), emergency visits, physician encounters (total and separately for specialists and general practitioners) and home care services (total and by type); (2) 1-year incremental costs attributable to becoming an HCU (total healthcare expenditures and by cost category) and (3) provincial budget impact of new senior HCUs in FY2013. Incremental healthcare use and costs were calculated as the difference between the two cohorts over 1-year period. They represent additional mean visits made or costs incurred by an HCU in the incident year compared with a non-HCU and the baseline year.

\section{Statistical analysis}

Baseline patient sociodemographic and health status characteristics of the two cohorts in FY2012 were compared using the absolute standardised difference (aSD), with aSD $>0.1$ indicating a meaningful difference. ${ }^{35}$ We then described the HCU cohort in the context of cost categories and their contribution to the HCU status by calculating the proportion (\%) of HCU in each cost category. Since we expected hospitalisations to be a frequent cause of new HCU status, we repeated this analysis for HCUs who were not hospitalised during the incident year to evaluate the contributions of cost drivers other than hospital admission. This was followed by a longitudinal comparison of the unadjusted healthcare use and costs in both cohorts for both the incident year and the preceding year.

Incremental healthcare use and costs were estimated using the recycled predictions method. ${ }^{36-39}$ Commonly used to evaluate the marginal effect of a covariate on the response variable, the method uses fitted regression models to predict incremental values of the outcomes in two hypothetical populations: one where all subjects are HCU and another where all are non-HCU, all the other covariates being the same. The difference in predicted means between the two populations indicates the incremental value. The method allows for correlation between outcome values in the year before the index year (FY2012) and after the index year (FY2013), while comparing HCU with non-HCU. CIs of the incremental values were obtained through the percentile method: random bootstrap resampling with 1000 iterations created a distribution where the 2.5th and 97.5 th percentiles were the $95 \%$ lower and upper bound CIs, respectively. ${ }^{39}$

We used generalised linear regression to model the study outcomes. Costs were modelled with gamma distribution and log-link function to handle the right-skewed data. ${ }^{4041}$ The choice of gamma distribution was confirmed by the modified Park test. ${ }^{42}$ For count data (eg, hospital admissions or home care visits), a negative binomial (NB) distribution was specified as the leading option to better account for overdispersion (ie, observed variance is greater than the assumed variance) ${ }^{43}{ }^{44}$ In cases of an NB model not converging, Poisson distribution was used. For both costs and count data, we used two-part models (Hurdle regression) to manage zero values in the response variables: the first part used a logistic regression to predict the probability of positive values of the outcome, while a gamma or an NB model was applied in the second 


\begin{tabular}{|c|c|c|c|}
\hline Characteristic & HCU (n=175 847) & Non-HCU ( $n=527$ 541) & aSD \\
\hline \multicolumn{4}{|l|}{ Sociodemographics } \\
\hline Age, mean (SD), year & $77.7 \pm 7.7$ & $77.7 \pm 7.7$ & 0 \\
\hline Sex, female, $(\%)$ & $93119(53)$ & $279501(53)$ & 0 \\
\hline Rural Index of Ontario score, mean (SD) & $12.2 \pm 18.2$ & $11.8 \pm 18.2$ & 0.02 \\
\hline Low income, $(\%)$ & $31843(18.1)$ & $92566(17.5)$ & 0.01 \\
\hline \multicolumn{4}{|l|}{ Health status } \\
\hline Adjusted diagnostic groups, mean (SD) $†$ & $10.2 \pm 4.0$ & $7.9 \pm 4.5$ & 0.54 \\
\hline Hypertension, $(\%)^{\star}$ & $110692(63.0)$ & $282867(53.6)$ & 0.19 \\
\hline Congestive heart failure, $(\%) \dagger$ & 25195 (14.3) & $36877(7.0)$ & 0.24 \\
\hline Chronic obstructive disease pulmonary, (\%)† & $48738(27.7)$ & $96513(18.3)$ & 0.23 \\
\hline Diabetes, $(\%) \dagger$ & 62014 (35.3) & $138794(26.3)$ & 0.2 \\
\hline Myocardial infarction, (\%)† & $12892(7.3)$ & $24024(4.6)$ & 0.12 \\
\hline Rheumatoid arthritis, (\%)† & $5607(3.2)$ & $9334(1.8)$ & 0.09 \\
\hline Malignancy, $(\%)^{\star}$ & 56855 (32.3) & $123932(23.5)$ & 0.2 \\
\hline Mental health condition, $(\%)^{*}$ & 67441 (38.4) & 144377 (27.4) & 0.24 \\
\hline
\end{tabular}

aSD with $\mathrm{aSD}>0.1$ indicating meaningful difference between $\mathrm{HCU}$ and non-HCU.

${ }^{*}$ Constructed based on expanded diagnosis codes.

†ICES-derived cohort.

aSD, absolute standardised difference; HCU, high-cost user; ICES, Institute for Clinical Evaluative Sciences.

stage for positive costs and counts, respectively. ${ }^{43}$ All the models were adjusted for previous resource use (eg, costs or healthcare use in FY2012), age, sex, ADGs and low-income status. Because our dataset included all senior HCU subjects in the province at the time of the study, we were able to estimate the total provincial public healthcare expenditures attributable to HCU status among Ontario seniors by multiplying the total incremental costs by the total number of senior HCU. Statistical analyses were conducted using SAS V.9.4.3 (SAS Institute). The SAS scripts are available as online supplemental material.

\section{Patient and public involvement}

Patients or public were not involved in the design of this retrospective cohort study.

\section{RESULTS}

\section{Patient characteristics}

The total study population consisted of 703388 seniors, of which 175847 were incident HCUs. This population of incident HCUs represents $46 \%$ of all HCUs in FY2013 ( $\mathrm{n}=383257$ ) but only $9.4 \%$ of the Ontario senior population and $1.4 \%$ of the total population in the province. ${ }^{20}$ As expected, the mean ages of the HCU and non-HCU cohorts were identical at 77.7 years (SD 7.7); $53 \%$ were women; and most resided in suburban areas (12.2 vs 11.8 , $\mathrm{aSD}=0.02$ ) (table 1). Compared with non-HCUs, HCUs had poorer health status as defined by both the number of aggregated diagnosis groups $(10.2$ vs $7.9, \mathrm{aSD}=0.54)$ and higher prevalence of chronic diseases. A relatively greater percentage of HCU cohort members had a primary care provider $(97 \%$ vs $88.6 \%$, aSD $=0.33)$.

\section{HCU status}

The 5\% HCU annual threshold for this study was \$10 192. As shown in figure 1, patients could become HCU through different combinations of incurred costs. Approximately $40 \%$ of the HCU became an HCU (ie, incurred at least $\$ 10192$ in total annual healthcare expenditures) due to a single cost category, predominantly hospital admissions $(70.1 \%)$. For $13 \%$ of the HCUs, more than one cost category was above the threshold (eg, hospital admission and rehabilitation costs). Among the remaining $47 \%$, no single cost category was sufficient to meet the expenditure threshold for HCU status: HCU status was achieved through expenditures in several cost categories. In this case, the most common contributing categories were physician compensation, drug benefits and hospitalisation.

As many as $11.7 \%(n=20501)$ of the HCU were not hospitalised during the incident year (online supplementary appendix 1). Their new HCU status was mainly due to a combination of physician compensation (99.8\%), ODB $(99.4 \%)$ and laboratory test costs $(87.3 \%)$, home care $(54.1 \%)$ and ED visits $(45.3 \%)$. Of note, some of the patients within several cost categories had costs high enough for the patient to become an HCU. Examples include $72.3 \%$ of patients in long-term care, $63.4 \%$ of patients with cancer care and $19.1 \%$ of patients with drug costs.

\section{Dynamics of change in healthcare use and costs}

Analysis of observed healthcare utilisation in the two cohorts identifies an upward trajectory in health services consumption among senior HCU. As shown in figure 2, 


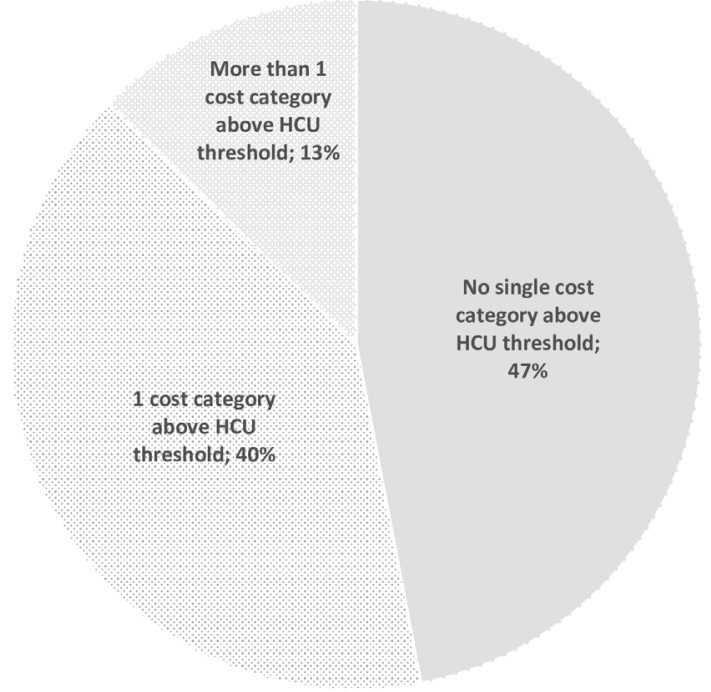

Figure 1 Proportion of new high-cost users (HCUs) that made the HCU threshold due to various types of costs. The graph presents the proportion of senior $\mathrm{HCU}$ in the context of cost categories that reached the HCU threshold of $\$ 10$ 192. One cost category (eg, hospital costs) reached the HCU threshold among $40 \%$ of new HCUs (per cent of patient in top five categories: hospital $(70.7 \%)$, cancer (8.1\%), ODB (7.3\%), LTC (5.1\%), HC (3.3\%)). More than one cost category (eg, hospital and physician costs) reached the $\mathrm{HCU}$ threshold among $13 \%$ of HCUs (per cent of patient in top five categories: hospital (95.1\%), physician (35.5\%), rehab (27.8\%), CCC (18.6\%), HC (13.6\%)). No single cost category reached the HCU threshold among $47 \%$ of new HCUs (per cent of patient in top five categories: physician $(99.9 \%$, mean \$3022), ODB (99.6\%, mean \$2127), hospital (88.7\%, mean $\$ 5611)$, laboratory $(87.1 \%$, mean $\$ 190)$, ED $(70 \%$, mean \$654). CCC, complex continuing care; ED, emergency department; HC, home care; LTC, long-term care; ODB, Ontario Drug Benefit.

compared with non-HCU, the HCU consumed more services in the preincident year across all care categories: physician encounters (mean per patient: 15.4 vs 10.1, $\mathrm{aSD}=0.55$ ), home care visits (mean per patient: 7.7 vs 1.8 ; $\mathrm{aSD}=0.24$ ), $\mathrm{ED}$ visits (mean per patient: 0.6 vs 0.3 ; $\mathrm{aSD}=0.26$ ) and hospital admissions (mean per patient: 0.04 vs 0.02 ; $\mathrm{aSD}=0.08$ ). This was followed by a dramatic increase in healthcare use among senior HCU during FY2013, while the service consumption among non-HCU remained relatively unchanged.

Similarly, the total public healthcare expenditures among senior HCU were higher in the preincident year compared with non-HCU (mean per patient: \$4166 vs $\$ 2372$, aSD $=0.74$ ), followed by a substantial spike during the incident year ( $\$ 29784$ vs $\$ 2471$; $\mathrm{aSD}=1.33$ ) (figure 3). While the major drivers of total costs were analogous in the two cohorts in the year before (in descending order: drug benefits, physician costs, hospital admissions or home care), the top contributors in the HCU cohort changed during the incident year. With an annual mean of 1.07 of hospital admissions (mean TLOS: 8.8 (SD 14.8)) among senior

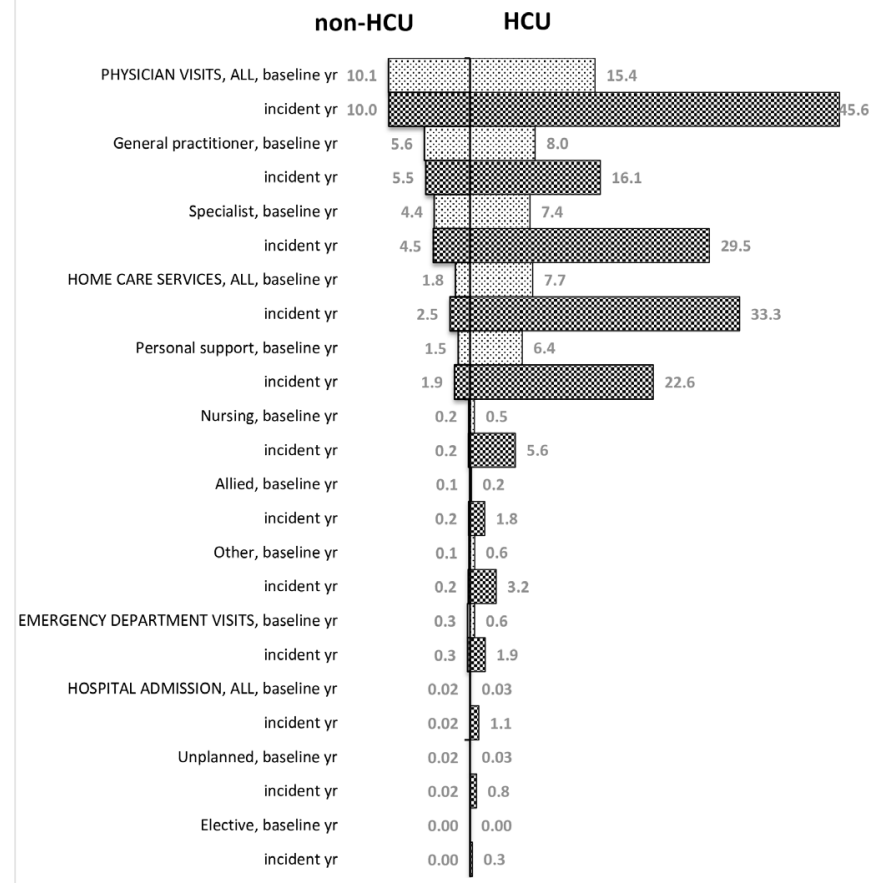

Figure 2 Dynamics of change in annual healthcare use, before (baseline) and during incident year, by HCU status and cost categories (mean per patient). The araph shows

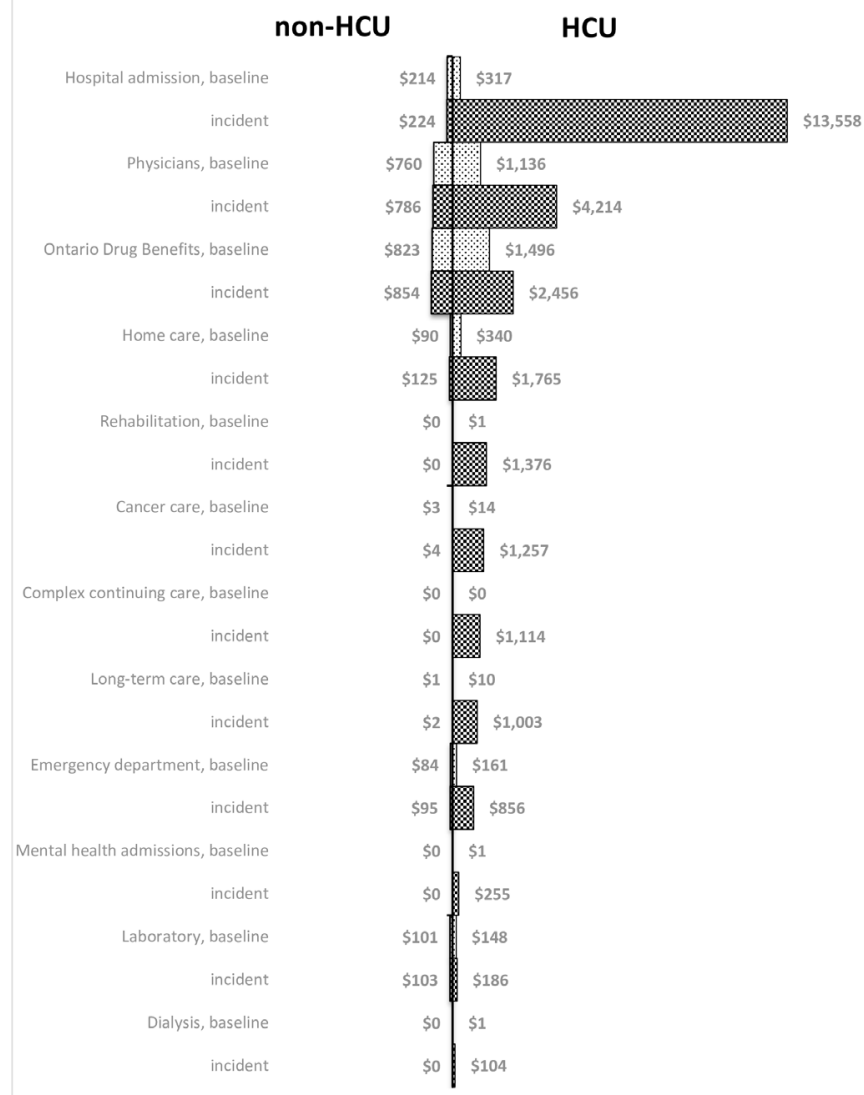

Figure 3 Dynamics of change in annual healthcare care expenditures before and after index year, by HCU status and cost categories (annual, mean per patient). total costs per patient (mean) among HCUs: $\$ 4166$ (baseline year) and $\$ 29$ 784 (incident year). Total costs per patient (mean) among non-HCUs: \$2372 (baseline year) and \$2471 (incident year). $\mathrm{HCU}$, high-cost user. 
Table 2 Incremental healthcare use associated with HCU status, by healthcare type

\begin{tabular}{lc}
\hline Healthcare type & $\begin{array}{l}\text { Annual incremental } \\
\text { utilisation, mean } \\
(\mathbf{9 5 \%} \mathbf{C l})\end{array}$ \\
\hline Hospital admission, all & $1.04(1.04$ to 1.05$)$ \\
\hline Hospital admission, elective & $0.29(0.29$ to 0.3$)$ \\
\hline Hospital admission, unplanned & $0.77(0.77$ to 0.78$)$ \\
\hline Emergency department visits & $1.4(1.4$ to 1.4$)$ \\
\hline Physician visits, all & $32.1(31.9$ to 32.3$)$ \\
\hline General practitioner visits & $9.3(8.7$ to 9.5$)$ \\
\hline Specialist visits & $22.8(22.7$ to 22.9$)$ \\
\hline Home care services, all & $25.1(24.4$ to 25.7$)$ \\
\hline Personal support & $15.6(15.3$ to 15.9$)$ \\
\hline Nursing & $5.3(4.9$ to 6.0$)$ \\
\hline Allied & $1.5(1.5$ to 1.6$)$ \\
\hline Other $\dagger$ & $2.8(2.7$ to 2.9$)$ \\
\hline
\end{tabular}

Annual incremental utilisation is an additional mean number of services received by an $\mathrm{HCU}$ in the incident year compared with a non-HCU and the baseline year.

${ }^{*}$ Fit using Poisson distribution; all other are fit using negative binomial.

†'Other' includes social services, case management and respite care.

$\mathrm{HCU}$, high-cost user.

HCU compared with a mean of 0.03 admissions (mean TLOS: 2.8 (SD 9.6)) for non-HCUs in FY2013, prolonged hospitalisations were the major driver of total healthcare expenditures $(\$ 13558)$ in the incident year. These were followed by physician (\$4214) and ODB costs (\$2456). In categories such as rehabilitation, complex continuing care, dialysis and mental health admissions, the costs incurred by senior HCUs at baseline and non-HCUs across both years were approximating zero: these categories were almost exclusively associated with the HCU status. Little change in the list of major cost drivers and the trajectory of costs over time was noticeable among non-HCU seniors. More detail is provided in online supplementary appendices 2 and 3 .

\section{Incremental costs and healthcare use}

Table 2 shows the magnitude of incremental healthcare use by senior HCU during the incident year adjusting for the preincident values and other covariates. Compared with the year before becoming an HCU, unplanned hospitalisations accounted for $74 \%$ of all incremental admissions at an additional mean of 0.77 hospitalisations per HCU (95\% CI 0.77 to 0.78 ) annually. Similarly, specialist visits constituted $75 \%$ of the incremental physician encounters at an additional mean of 22.8 visits $(95 \%$ CI 22.7 to 22.9), whereas personal support worker visits contributed the most to the incremental home care use at additional mean of 15.6 visits (95\% CI 15.3 to 15.9 ) per HCU patient.

The total annual mean adjusted costs attributable to HCU status were \$25 527 (95\% CI \$25 383 to \$25 670)
Table 3 Incremental expenditures associated with $\mathrm{HCU}$ status, by cost component and total

\begin{tabular}{ll}
\hline Cost component & $\begin{array}{l}\text { Annual incremental costs*, } \\
\text { mean (95\% Cl) }\end{array}$ \\
\hline Hospital admission & $\$ 13428(13334$ to 13534$)$ \\
\hline Physicians & $\$ 3150(3134$ to 3168$)$ \\
\hline Outpatient drug benefits & $\$ 1493(1462$ to 1523$)$ \\
\hline Rehabilitation & $\$ 1430(1392$ to 1467$)$ \\
\hline Home care & $\$ 1363(1347$ to 1378$)$ \\
\hline Cancer care & $\$ 1226(1200$ to 1253$)$ \\
\hline Complex continuing care & $\$ 1213(1168$ to 1257$)$ \\
\hline Long-term care & $\$ 1021(995$ to 1046$)$ \\
\hline Emergency department & $\$ 684(679$ to 687$)$ \\
\hline Mental health admissions & $\$ 258(238$ to 278$)$ \\
\hline Dialysis & $\$ 89(79$ to 99$)$ \\
\hline Laboratory tests & $\$ 51(50$ to 52$)$ \\
\hline Total incremental cost & $\$ 25527(25383$ to 25670$)$ \\
\hline
\end{tabular}

Annual incremental costs are additional mean expenditures incurred by an $\mathrm{HCU}$ in the incident year compared with a non-HCU and the baseline year.

${ }^{*}$ Costs were modelled to follow gamma distribution with log-link function.

(table 3), with hospital admissions being by far the major contributor at an additional mean of \$13 428 (95\% CI $\$ 13333$ to $\$ 13533$ ) per HCU. Details of the regression analyses are provided in online supplementary appendices 4 and 5. Given the size of the senior incident HCU population ( $\mathrm{n}=175847)$, the estimated provincial budget impact of the senior incident HCU status was $\$ 4.5$ billion (CAD). This accounts for approximately $9 \%$ of the 2013 total provincial healthcare expenditures ( $\$ 51$ billion). ${ }^{46}$

\section{DISCUSSION}

The study has examined a cohort of new senior HCU patients compared with matched non-HCUs focusing on the absolute and incremental comparative healthcare use and expenditures before and after HCU conversion. We determined that although senior HCUs were already on an upward trajectory during the year before HCU status, showing higher healthcare utilisation and costs in the preincident year, the HCU status was associated with a spike in healthcare expenditures. We found that seniors became HCU through incurring costs in various combinations, although half of the senior HCU could reach the HCU status by incurring costs from only one or two categories, mainly prolonged hospitalisation. Approximately $12 \%$ of HCUs had no hospitalisation in the incident year: they achieved HCU status by incurring costs largely on physician services and prescription medications. Compared with non-HCU, senior HCU incurred an additional \$25 527 per patient in total incremental public healthcare expenditures and cost almost $1 / 10$ of the 
provincial budget in the incident year. Hospitalisations, physician compensation and ODB were responsible for the highest incremental costs.

This study fills a current gap in the HCU economic literature, especially Canadian HCU studies where few of them have focused on seniors or used a comparative group of non-HCUs. Also, as opposed to cross-sectional studies that are common in the area of HCU research, we were able to capture the economic burden attributable to HCU status among senior Ontarians using longitudinal data. Our approach of the recycled predictions has allowed us to compare the healthcare use and costs between HCUs and a matched cohort of non-HCUs while taking account of the correlation between the prevalues and postvalues, managing excessive zero values by developing two-part models, and adjusting for confounding by including important sociodemographic and health status covariates in the models. Another option we considered was the difference-in-differences estimator. ${ }^{47} 48$ Frequently employed by economists to assess the impact of introducing a policy or a change in the system, its use is, however, conditional on two major assumptions that need to be met: parallel trends and no group variation at baseline. While the latter could be dealt with using statistical adjustment, the former assumes that trajectories in outcomes (ie, costs and use) between the groups are the same prior to the exposure (ie, HCU conversion). Because we only had access to 1 year of data prior to the incident year (ie, the baseline year) by design, it was not possible to determine the trajectories between the cohorts.

Consistent with nine studies of senior HCUs identified by Wammes et al, our results confirm the high burden of common conditions among senior HCUs, the important impact of inpatient care costs, the increasing role of home and long-term care in the HCU cost profile. Some studies also mention non-hospitalised senior HCUs without providing their detailed description. ${ }^{1049}$ Our findings are, however, challenging to compare with these for several reasons. First, in addition to the incremental values, we provide a comprehensive assessment of costs and healthcare utilisation for a specific segment of the HCU population: senior incident cases. To our knowledge, no other studies have examined this specific patient population, especially in such detail. ${ }^{5}$ Second, as Wammes et al show, the HCU threshold used in the USA and other countries (eg, Denmark and Germany) is often $10 \%$, while Canadian studies commonly apply the $5 \%$ threshold. ${ }^{5}$ Third, the spectrum of cost categories included in analysis may vary between countries and even provinces in Canada. Prescription drug costs, for example, the source of one of the highest incremental values in our study, were not covered by the US Medicare program (which covers senior patients) until 2003, although the launch of a fully developed program was delayed until mid-2000s,${ }^{50}{ }^{51}$ limiting the comparability of earlier studies that relied only on Medicare payments. ${ }^{11235}$ In this respect, our efforts to standardise cost analyses by using a costing methodology that allows obtaining patient-level expenditures from multiple sources in one standard way is a step towards higher comparability of future studies.

\section{Strengths and limitations}

Our study has several strengths. First, the study is population based, including all incident senior HCU in the province. Second, the study examines incident HCU, which provides important information on the driving factors for HCU status. Third, we included a comprehensive spectrum of the most important cost categories that contribute to total public healthcare expenditures in the province.

The study also has important limitations. The nature of methodology applied to calculate the costs was different across various cost categories. As opposed to the nominal costs per visit (eg, physician or home care) or prescription claim, some of the costs were estimations, or example, a provincial average cost per case of inpatient care weighted for resource intensity. ${ }^{34}$ However, when used for comparisons at a provincial level, these estimations are considered acceptable.$^{34}$ Also, despite our comprehensive coverage of cost categories, some public healthcare expenditures are not accounted for. Examples include community services (eg, community services for elderly) and public health costs. In addition, a few of the cost categories included the analysis may not be captured in full. Most notably, we did not have access to the costs of outpatient intravenous chemotherapy, which can be costly. ${ }^{53}$ Also, long-term care residents pay a portion of the costs out of pocket. ${ }^{54}$ Despite these limitations, it is unlikely that the unaccounted costs for individual healthcare services amount to more than $5 \%-8 \%$ of total public expenditures on healthcare. ${ }^{10}{ }^{18}$ At the same time, the true hospitalisation expenses may be underestimated as physician billings for inpatient services are currently captured by a separate cost category which makes our estimates of the hospital costs conservative. Further, different HCU thresholds may yield different estimations of the incremental costs. Although ours is the most commonly used HCU threshold in Canada, ${ }^{5}$ our findings are largely comparable to studies with the same threshold and the choice of cost categories. Finally, since our study by design focuses on incident senior HCUs, we did not examine other senior HCU population groups such as prevalent HCUs (ie, those who have been HCU both in FY2012 and FY2013) or those individuals who were HCUs in FY2012 but not in FY2013.

Despite these limitations, our findings have policy and research implications. There is currently no clear internationally accepted definition of the HCU. ${ }^{7}$ They are also referred to by many names (eg, heavy, frequent or high needs users) that are used interchangeably with HCU. ${ }^{7}$ However, our data show that frequent users of healthcare may not be synonymous with HCUs of healthcare and both need to be distinguished. One prolonged hospital stay, for example, can drive a senior patient to become an HCU. Although interventions have been introduced to either prevent or divert such hospitalisations, their success is unclear. ${ }^{7}$ Further efforts are needed to examine 
predictors at the prehospital level and to identify actionable cost drivers during admission. ${ }^{55}$ At the same time, more than 1/10 of senior HCUs had no hospital costs. The latter subset of HCUs requires further investigation. Reducing ODB expenditures by exploring pharmaceutical policy or pricing strategies (eg, generic drug tendering) stands out as a promising but challenging area to achieve potential cost reductions ${ }^{56}$ Canada has recently made steps to alleviate the burden of drug costs by negotiating lower prices of generic and non-generic drugs with manufacturers. ${ }^{57}$ Although there may be room for further savings among generic drugs, ${ }^{58}$ these may be offset by the growing share of expensive biologics coupled with just a modest uptake of biosimilars. ${ }^{59}$ Finally, future cost analysis of senior HCUs could benefit from greater data granularity. Following a patient longitudinally by type of care received in the incident year and time of death, for example, it may be possible to more precisely identify the point of HCU conversion, differentiate between outpatient and inpatient costs that contribute to it, and allocate costs more with greater accuracy, including specific clinical conditions (eg, cognitive impairment among seniors) or conducting joint cost-survival modelling. ${ }^{6061}$

\section{CONCLUSION}

Costs attributable to incident senior HCU status accounted for almost $1 / 10$ of the provincial budget. Prolonged hospitalisations made a major contribution to the total incremental costs. However, categories such as physician billings, drug benefits and other, in various combinations, also were important. A subgroup of patients that became HCU without hospitalisation requires further investigation.

\section{Author affiliations}

${ }^{1}$ Health Research Methods, Evidence, and Impact, McMaster University Faculty of Health Sciences, Hamilton, Ontario, Canada

${ }^{2}$ Department of Health Research Methods, Evidence, and Impact, McMaster University Faculty of Health Sciences, Hamilton, Ontario, Canada

${ }^{3}$ Clinical Pharmacology \& Toxicology, St. Joseph's Healthcare, Hamilton, Ontario, Canada

${ }^{4}$ Département de médecine sociale et préventive, Faculté de Médecine, Université Laval, Quebec City, Quebec, Canada

${ }^{5}$ Department of Health Research Methods, Evidence, and Impact, McMaster University, Hamilton, Ontario, Canada

${ }^{6}$ ICES, Toronto, Ontario, Canada

${ }^{7}$ Li Ka Shing Knowledge Institute, Toronto, Ontario, Canada

${ }^{8}$ Health Research Methods, Evidence, and Impact, McMaster University, Toronto, Ontario, Canada

Contributors SM, J-ET, AH, JL, JMP, TG, LM and JRG conceptualised the study. SM, J-ET, AH, JL, JRG, LM, JMP, TG and PP contributed to its design. JMP, PP and TG were instrumental in creating datasets. PP provided assistance with data analysis. SM prepared the initial draft of the manuscript and revised it based on coauthors' feedback: J-ET, AH, JL, JMP, TG, JRG, LM and PP provided comments to the initial draft, further revisions, read and approved the final manuscript. The responsibility of study implementation lies with the principal investigator (SM) that is supported and supervised primarily by J-ET.

Funding This work is supported by in-kind support from the Ontario Drug Policy Research Network (ODPRN) and by personnel awards from the Canadian Institutes of Health Research (CIHR) Drug Safety and Effectiveness Cross-Disciplinary Training (DSECT) Program, the Program for Assessment of Technology in Health (PATH), The Research Institute of St Joe's Hamilton, St Joseph's Healthcare Hamilton and an Ontario Graduate Scholarship (OGS). The work also is supported by ICES, an independent research institute funded by the Ontario Ministry of Health and LongTerm Care (MOHLTC). ODPRN is funded by grants from the Ontario MOHLTC and the Ontario Strategy for Patient-Orientated Research (SPOR) Support Unit, which is supported by CIHR and the Province of Ontario. No endorsement by ICES or the Ontario MOHLTC is intended or should be inferred. Parts of this material are based on data and information compiled and provided by the Canadian Institute for Health Information (CIHI).

Disclaimer The opinions, results and conclusions reported in this article are those of the authors and are independent from the funding sources. The analyses, conclusions, opinions and statements expressed herein are those of the authors and not necessarily those of $\mathrm{CIHI}$.

Competing interests None declared.

Patient consent for publication Not required.

Ethics approval This study was approved by Hamilton Integrated Research Ethics Board (ID\#1715 C).

Provenance and peer review Not commissioned; externally peer reviewed.

Data availability statement The dataset from this study is held securely in coded form at the Institute for Clinical Evaluative Sciences (ICES). While data sharing agreements prohibit ICES from making the dataset publicly available, access may be granted to those who meet prespecified criteria for confidential access, available at http://www.ices.on.ca/DAS. The full dataset creation plan is available from the authors on request. Analytic codes (in SAS) used to conduct the analysis are available as online supplementary material.

Open access This is an open access article distributed in accordance with the Creative Commons Attribution Non Commercial (CC BY-NC 4.0) license, which permits others to distribute, remix, adapt, build upon this work non-commercially, and license their derivative works on different terms, provided the original work is properly cited, appropriate credit is given, any changes made indicated, and the use is non-commercial. See: http://creativecommons.org/licenses/by-nc/4.0/.

ORCID iD

Lawrence Mbuagbaw http://orcid.org/0000-0001-5855-5461

\section{REFERENCES}

1. World Health Organization. Global health expenditure database, 2000-2015. secondary global health expenditure database, 20002015, 2018. Available: http://apps.who.int/nha/database/ViewData/ Indicators/en

2. Canadian Institute for Health Information. National health expenditure trends, 1975 to 2017. Ottawa, ON: Canadian Institute for Health Information, 2017.

3. Pritchard D, Petrilla A, Hallinan S, et al. What contributes most to high health care costs? health care spending in high resource patients. J Manag Care Spec Pharm 2016;22:102-9.

4. Tamang S, Milstein A, Sørensen HT, et al. Predicting patient 'cost blooms' in Denmark: a longitudinal population-based study. BMJ Open 2017;7:e011580.

5. Wammes JJG, van der Wees PJ, Tanke MAC, et al. Systematic review of high-cost patients' characteristics and healthcare utilisation. BMJ Open 2018;8:e023113.

6. Bleich SN, Sherrod C, Chiang A, et al. Systematic review of programs treating High-Need and high-cost people with multiple chronic diseases or disabilities in the United States, 2008-2014. Prev Chronic Dis 2015;12:E197.

7. Lee JY, Muratov S, Tarride J-E, et al. Managing high-cost healthcare users: the International search for effective Evidence-Supported strategies. J Am Geriatr Soc 2018;66

8. Figueroa JF, Joynt Maddox KE, Beaulieu N, et al. Concentration of potentially preventable spending among high-cost Medicare subpopulations. Ann Intern Med 2017;167:706-13.

9. Joynt KE, Figueroa JF, Beaulieu N, et al. Segmenting high-cost Medicare patients into potentially actionable cohorts. Healthc 2017;5:62-7.

10. Wodchis WP, Austin PC, Henry DA. A 3-year study of high-cost users of health care. Can Med Assoc J 2016;188:182-8.

11. Lieberman SM, Lee J, Anderson T, et al. Reducing the growth of Medicare spending: geographic versus patient-based strategies. Health Aff 2003;22(Suppl1):W3-603-W3-613.

12. Ku L-JE, Chiou M-J, Liu L-F. Variations in the persistence of health expenditures and the implications for the design of capitation payments in Taiwan. $J$ Health Serv Res Policy 2015;20:146-53. 
13. Sinha SK. Why the elderly could bankrupt Canada and how demographic imperatives will force the redesign of acute care service delivery. Healthc Pap 2011;11:46-51. discussion 86-91.

14. Holtz-Eakin D. High-Cost Medicare beneficiaries secondary highcost Medicare beneficiaries, 2005. Available: http://www.cbo.gov/ publication $/ 16487$

15. Rais S, Nazerian A, Ardal S, et al. High-Cost users of Ontario's healthcare services. Hcpol 2013;9:44-51.

16. Reid R, Evans R, Barer M, et al. Conspicuous consumption: characterizing high users of physician services in one Canadian Province. J Health Serv Res Policy 2003;8:215-24.

17. Figueroa JF, Frakt $A B$, Lyon ZM, et al. Characteristics and spending patterns of high cost, non-elderly adults in Massachusetts. Healthc 2017;5:165-70.

18. Muratov S, Lee J, Holbrook A, et al. Regional variation in healthcare spending and mortality among senior high-cost healthcare users in Ontario, Canada: a retrospective matched cohort study. BMC Geriatr 2018;18:262.

19. Muratov S, Lee J, Holbrook A, et al. Senior high-cost healthcare users' resource utilization and outcomes: a protocol of a retrospective matched cohort study in Canada. BMJ Open 2017;7:e018488.

20. Canada S. Population by year, by Province and Territory. secondary population by year, by Province and territory, 2016. Available: http:// www.statcan.gc.ca/tables-tableaux/sum-som/101/cst01/demo02aeng.htm

21. CIHI. National Health Expenditure Trends, 1975 to 2016. Ottawa, ON Canadian Institute for Health Information, 2016.

22. Guilcher SJT, Bronskill SE, Guan J, et al. Who are the high-cost users? A method for person-centred Attribution of health care spending. PLoS One 2016;11:e0149179.

23. Riley GF. Long-Term trends in the concentration of Medicare spending. Health Aff 2007;26:808-16.

24. Ontario Ministry of Health and Long-term Care. Understadning Ontario's drug programs. secondary Understadning Ontario's drug programs, 2018. Available: http://www.health.gov.on.ca/en/public/ programs/drugs/

25. LHINs Ontario's. Ontario's local health integration networks. secondary Ontario's local health integration networks, 2017. Available: www.lhins.on.ca

26. Kralj B. Measuring 'rurality' for purposes of health-care planning: an empirical measure for Ontario. Ontario Medical Review, 2000: 33-52.

27. Johns Hopkins ACG. System version 10.0. technical reference guide. secondary technical reference guide; 2014.

28. Austin PC, van Walraven C, Wodchis WP, et al. Using the Johns Hopkins aggregated diagnosis groups (ADGS) to predict mortality in a general adult population cohort in Ontario, Canada. Med Care 2011:49:932-9.

29. Gershon AS, Wang C, Guan J, et al. Identifying individuals with physcian diagnosed COPD in health administrative databases. COPD 2009;6:388-94.

30. Schultz SE, Rothwell DM, Chen Z, et al. Identifying cases of congestive heart failure from administrative data: a validation study using primary care patient records. Chronic Dis Inj Can 2013;33:160-6.

31. de Oliveira C, Cheng J, Vigod S, et al. Patients With High Mental Health Costs Incur Over 30 Percent More Costs Than Other HighCost Patients. Health Aff 2016;35:36-43.

32. Joynt KE, Gawande AA, Orav EJ, et al. Contribution of preventable acute care spending to total spending for high-cost Medicare patients. JAMA 2013;309:2572-8.

33. Stanton MW RM. High concentration of US health expenditures. Research in Action: Agency for Healthcare Research and Quality, 2006.

34. Wodchis WP, Bushmeneva K, Nikitovic M, et al. Guidelines on person-level costing using administrative databases in Ontario. Working paper series. Toronto: Health System Performance Research Network, 2013

35. Mamdani M, Sykora K, Li P, et al. Reader's guide to critical appraisal of cohort studies: 2. assessing potential for confounding. BMJ 2005;330:960-2.

36. Basu A, Arondekar BV, Rathouz PJ. Scale of interest versus scale of estimation: comparing alternative estimators for the incremental costs of a comorbidity. Health Econ 2006;15:1091-107.
37. Chang C, Lee SM, Choi BW, et al. Costs attributable to overweight and obesity in working asthma patients in the United States. Yonsei Med J 2017;58:187-94.

38. Lange A, Zeidler J, Braun S. One-Year disease-related health care costs of incident vertebral fractures in osteoporotic patients. Osteoporos Int 2014;25:2435-43.

39. Mannino DM, Higuchi K, Yu T-C, et al. Economic burden of COPD in the presence of comorbidities. Chest 2015;148:138-50.

40. Gregori D, Petrinco M, Bo S, et al. Regression models for analyzing costs and their determinants in health care: an introductory review. Int J Qual Health Care 2011;23:331-41.

41. Basu A, Manning WG, Mullahy J. Comparing alternative models: log vs COX proportional hazard? Health Econ 2004;13:749-65.

42. Manning WG, Mullahy J. Estimating log models: to transform or not to transform? J Health Econ 2001;20:461-94.

43. Lui WS, Cela J. Count data models in SAS. secondary count data models in SAS, 2008. Available: http://www2.sas.com/proceedings/ forum2008/371-2008.pdf

44. Elhai JD, Calhoun PS, Ford JD. Statistical procedures for analyzing mental health services data. Psychiatry Res 2008;160:129-36.

45. Mihaylova B, Briggs A, O'Hagan A, et al. Review of statistical methods for analysing healthcare resources and costs. Health Econ 2011;20:897-916.

46. Institute for Competitiveness \& Prosperity. Building better health care: policy opportunities for Ontario. Policy opportunities for Ontario: Secondary Building Better Health Care, 2014. https://www. competeprosper.ca/uploads/WP20_BetterHealthCare_FINAL.pdf

47. Stock JH, Watson MW. Introduction to Econometrics, 2011.

48. Dimick JB, Ryan AM. Methods for evaluating changes in health care policy: the difference-in-differences approach. JAMA 2014;312:2401-2.

49. Lee NS, Whitman N, Vakharia N, et al. High-Cost Patients: Hot-Spotters Don't Explain the Half of It. J Gen Intern Med 2017;32:28-34.

50. Oliver TR, Lee PR, Lipton HL. A political history of Medicare and prescription drug coverage. Milbank Q 2004;82:283-354.

51. Zhang Y, Donohue JM, Newhouse JP, et al. The effects of the coverage gap on drug spending: a closer look at Medicare Part D. Health Aff 2009;28(Supplement 1):w317-25.

52. Ganguli I, Thompson RW, Ferris TG. What can five high cost patients teach us about healthcare spending? Healthc 2017;5:204-13.

53. de Oliveira C, Bremner KE, Pataky R, et al. Understanding the costs of cancer care before and after diagnosis for the 21 most common cancers in Ontario: a population-based descriptive study. CMAJ Open 2013;1:E1-E8.

54. Ontario Long Term Care Association. About long-term care in Ontario: Facts and figures. Secondary Ontario Long Term Care Association - About long-term care in Ontario: Facts and figures, 2019. Available: https://www.oltca.com/oltca/OLTCA/Public/ LongTermCare/FactsFigures.aspx

55. Muratov S, Lee J, Holbrook A, et al. Unplanned index hospita admissions among new older high-cost health care users in Ontario: a population-based matched cohort study. CMAJ Open 2019;7:E537-E545.

56. Morgan S, Persaud N. Generic drug prices could drop - if rebates are replaced with public tendering. secondary generic drug prices could drop - if rebates are replaced with public tendering. Available: https://healthydebate.ca/opinions/generic-drug-prices

57. The Council of the Federation. The pan-Canadian pharmaceutical alliance, 2018. Available: http://www.canadaspremiers.ca/pancanadian-pharmaceutical-alliance/

58. Morgan SG, Persaud N. New generic pricing scheme maintains high prices and risks of shortages. Can Med Assoc J 2018;190:E41 $0-E 411$.

59. Lungu E, Warwick G. Potential savings from biosimilars in Canada. secondary potential savings from biosimilars in Canada. Available: https://www.cadth.ca/sites/default/files/symp-2017/presentations/ april24-2017/Concurrent-Session-B4-Gary-Warwick.pdf

60. Huang Y. Cost analysis with censored data. Med Care 2009;47(7 Suppl 1):S115-S119.

61. Wijeysundera HC, Wang X, Tomlinson G, et al. Techniques for estimating health care costs with censored data: an overview for the health services researcher. Clinicoecon Outcomes Res 2012;4:145-55 